\title{
Evaluación de la ingesta proteica y la actividad física asociadas con la sarcopenia del adulto mayor
}

\author{
Gloria Gabriela Peña-Ordóñez ${ }^{a}$, Lilia Patricia Bustamante-Montes ${ }^{a,{ }^{*}},{ }^{2}$ Ninfa Ramírez-Duran ${ }^{a}$, \\ Elizabeth Halley-Castillo ${ }^{b}$, Ligia García-Cáceres
}

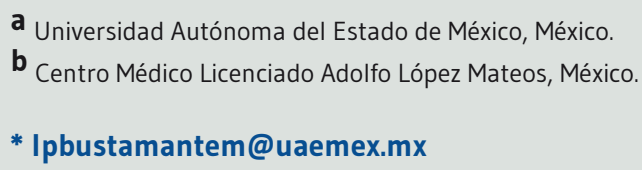

Recibido el 15 de agosto de 2015; aceptado el 28 de octubre de 2015.

\section{PALABRAS CLAVE}

Sarcopenia;

Ingesta proteica;

Ejercicio;

Actividad física.
Evaluación de la ingesta proteica y la actividad física asociadas con la sarcopenia del adulto mayor

\section{RESUMEN}

Introducción: Los adultos mayores son un grupo vulnerable y se refleja fácilmente en su estado nutricional, la mayoría no cubre sus requerimientos nutricionales y son inactivos físicamente. Una ingesta proteica menor a $1,0 \mathrm{~g} / \mathrm{kg}$ de peso/día y un bajo nivel de actividad física $(<3,5$ MET) son factores asociados a sarcopenia. El objetivo del estudio es determinar la asociación entre la ingesta proteica y actividad física con sarcopenia del adulto mayor.

Material y Métodos: Estudio observacional, analítico, prospectivo, de casos y controles. Se realizó muestreo por conveniencia en pacientes mayores de 60 años del servicio de consulta externa del Centro Médico Adolfo López Mateos de Toluca, México. Se aplicaron cuestionarios para determinar la ingesta proteica, actividad física y pruebas diagnósticas para sarcopenia (porcentaje de masa muscular, fuerza manual y velocidad de marcha). Se reclutaron 115 sujetos pero se incluyeron 110 (55 casos y 55 controles).

Resultados: Se obtuvo la razón de momios de las variables estudiadas, encontrándose que por cada gramo de ingesta de proteína total se reduce un $3 \%$ el riesgo de sarcopenia y por cada unidad del porcentaje de grasa se incrementa el riesgo un $20 \%$. No se encontró diferencia estadísticamente significativa en la actividad física, existe homogeneidad entre casos y controles respecto a los MET consumidos.

Conclusiones: La ingesta proteica es un factor protector contra la sarcopenia y una excesiva acumulación de grasa es un factor de riesgo para padecerla. Es importante seguir investigando la relación entre ambas en adultos mayores. 
Evaluation of protein intake and physical activity associated with sarcopenia in the elderly

\section{KEYWORDS}

Sarcopenia;

Dietary proteins;

Exercise;

Motor activity.

\section{ABSTRACT}

Introduction: The aim of this study was to determine the association between protein intake and physical activity with sarcopenia of the elderly. Older people are a vulnerable group and are easily reflected in their nutritional status, most do not cover their nutritional requirements and are physically inactive. A protein intake $<1.2 \mathrm{~g} / \mathrm{kg} /$ day and a low level of physical activity ( $<3.5 \mathrm{MET}$ ) are factors associated with sarcopenia.

Material and Methods: Observational, analytical, prospective, case-control study. Sampling was done for convenience in patients over 60 years of service outpatient Medical Center Adolfo Lopez Mateos, Toluca, Mexico. Questionnaires were used to determine protein intake and physical activity, and diagnostic tests for Sarcopenia (percentage of muscle mass, strength and speed Manual operation). 115 subjects were enrolled but 110 ( 55 cases and 55 controls) were included.

Results: The odds ratio (OR) of the variables was obtained, finding that for every gram of total protein intake of $3 \%$ reduces the risk of sarcopenia and per unit of percent fat increases the risk by $20 \%$. No statistically significant difference was found in physical activity, there is homogeneity between cases and controls regarding MET consumed.

Conclusions: Protein intake is a protective factor against sarcopenia and excessive accumulation of fat is a risk factor for this disorder. It is important to further investigate the relationship between the two in older adults.

CITA

Peña-Ordóñez GG, Bustamante-Montes LP, Ramírez-Duran N, Halley-Castillo E, García-Cáceres L. Evaluación de la ingesta proteica y la actividad física asociadas con la sarcopenia del adulto mayor. Rev Esp Nutr Hum Diet. 2016; 20(1): 16 - 22. DOI: 10.14306/renhyd.20.1.178

\section{INTRODUCCIÓN}

La sarcopenia es un síndrome geriátrico caracterizado por reducción grave, progresiva y generalizada de masa y fuerza muscular que afecta el rendimiento físico, predisponiendo a mala calidad de vida y muerte.

A nivel mundial, la prevalencia de sarcopenia oscila entre 5 y $32 \%$ de la población que tiene entre 60 y 70 años de edad, y de 11 a $80 \%$ en la que rebasa los 80 años ${ }^{1-3}$.

La clasificación según su progresión se compone de tres estadios propuestos por el "Grupo Europeo de Trabajo sobre Sarcopenia en Personas de Edad Avanzada" (EWGSOP por sus siglas en inglés): 1) presarcopenia: masa muscular deficiente como único criterio; 2) sarcopenia: masa muscular deficiente, fuerza deficiente o bajo rendimiento físico; 3) sarcopenia grave: los tres criterios están presentes ${ }^{3}$.

Estudios recientes han comparado la ingesta energética y nutricional en personas con baja y adecuada masa muscular, las de masa muscular baja tienen deficiencias de proteínas, hidratos de carbono y nutrientes inorgánicos. Es importante identificar deficiencias de proteínas de alto valor biológico porque son parte fundamental de los mecanismos vinculados a sarcopenia debido a la alteración en la homeostasis de síntesis de proteína muscular que promueve su degradación y limita la disponibilidad de aminoácidos esenciales para la síntesis proteica ${ }^{3}$.

A partir de los 70 años la pérdida de masa muscular es mayor al $15 \%$, por tanto disminuye la tasa metabólica en reposo, el apetito y la ingesta de alimentos, provocando deficiencias energético-proteicas. 
Los hábitos alimentarios de los adultos mayores son más heterogéneos que los del resto de la población. La cantidad y el tipo de alimentos que consumen son determinados por factores fisiológicos, psicosociales y económicos, estos repercuten en el estado nutricional. La desnutrición es debida a su desequilibrio y es difícil revertirla en personas de edad avanzada porque se asocia a una elevada tasa de morbilidad, prolongadas estancias hospitalarias y mayor mortalidad $^{4-7}$.

La "Ingesta Diaria Recomendada" (RDA por sus siglas en inglés) indicada para proteínas (0,8 a $1 \mathrm{~g} / \mathrm{kg}$ de peso/día) es insuficiente para población geriátrica porque su necesidad proteica aumenta ${ }^{8}$ y no es suficiente para sintetizar músculo, esto es objeto de debate en la comunidad científica ${ }^{9}$. La Sociedad de Medicina Geriátrica de la Unión Europea (EUGMS por sus siglas en inglés) y otras organizaciones recomiendan ingerir de 1,0 a 1,2 g de proteína/kg de peso/día para mantener la masa y función muscular, estas son mayores a las propuestas por la RDA, pero sugiere individualizar los requerimientos de una manera segura y tolerada. El tipo de proteína ingerida también influye en la síntesis, la proteína de alto valor biológico es más completa en aminoácidos esenciales, principalmente de leucina que es indispensable porque se metaboliza en células del músculo ${ }^{10,11}$, pero se requieren $14 \mathrm{mg} / \mathrm{kg}$ de peso al día ${ }^{12}$ que difícilmente cubren los adultos mayores.

Otro factor de riesgo es un bajo nivel de actividad física. La ausencia de ejercicio físico causa atrofia muscular, afectando las capacidades físicas (principalmente fuerza) y coordinativas.

El problema de un bajo nivel de actividad física (<3,5 MET) radica en el estilo de vida que se adquiere durante la juventud porque tiende a persistir en etapas posteriores, esto complica el proceso de envejecimiento porque las tasas de discapacidad por inactividad física son elevadas y las personas presentan dificultades para ejecutar actividades de la vida diaria ${ }^{13-15}$

\section{MATERIAL Y MÉTODOS}

Diseño de estudio: Observacional, analítico, de casos y controles incidentes de mayo de 2013 a febrero de 2014, realizado con pacientes del Centro Médico Adolfo López Mateos en Toluca, México. Una vez obtenida la aprobación del comité de ética, se reclutaron los sujetos por muestreo por conveniencia o no probabilístico.

Criterios de inclusión: Pacientes de 60 años o más, sanos o con enfermedades crónicas degenerativas controladas, que firmaron el consentimiento informado (basado en la Ley General de Salud de México y Declaración de Helsinki).

Criterios de exclusión: Sujetos con discapacidad física, enfermedad neurológica relacionada con la memoria, párkinson, cáncer, insuficiencia cardiaca o renal y con marcapasos.

Criterios de eliminación: Se eliminaron 5 sujetos que no completaron cuestionarios y pruebas diagnósticas.

\section{Procedimiento y Recolección de datos}

1) Aplicación de cuestionarios: Se aplicó a cada sujeto, encuesta dietética de frecuencia de consumo de alimentos de la Encuesta Nacional de Salud y Nutrición (ENSANUT) para estimar ingesta proteica y cuestionario de actividad física para adultos mayores CHAMPS.

2) Pruebas de diagnóstico para Sarcopenia: Se realizaron conforme el algoritmo propuesto por el EWGSOP. Se realizó prueba de velocidad de la marcha en $6 \mathrm{~m}$ lineales cronometrados $(\leq 0,8 \mathrm{~m} / \mathrm{s}$ bajo rendimiento físico y $>0,8 \mathrm{~m} / \mathrm{s}$ rendimiento físico adecuado)

Se realizó prueba de fuerza manual, con dinamómetro digital de agarre ajustable Takei 5101, alentando a los sujetos a hacer el máximo esfuerzo durante la presión (baja fuerza $<30 \mathrm{~kg}$ en varones y $<20 \mathrm{~kg}$ en mujeres). Cada sujeto realizó dos ejecuciones en la mano dominante tomando el valor máximo.

La masa muscular (kg y porcentaje, bajo en mujeres $<36 \%$, hombres $<40 \%$ ) se midió con un impedanciómetro modelo InBody R20 (Tetra-polar con 8 electrodos táctiles y frecuencia de 20 a $100 \mathrm{kHz}$ ). Se pidió el mínimo de ropa posible y el despojo de objetos metálicos.

De acuerdo con los resultados obtenidos se clasificaron en casos y controles.

Análisis estadístico: Los datos fueron capturados por duplicado en paquete estadístico STATA 11; se realizó análisis exploratorio para limpieza de datos y corrección de los mismos contra cuestionarios, análisis descriptivo de la población bajo estudio utilizándose medidas de tendencia central, dispersión y porcentajes entre variables (cuantitativas y cualitativas). También se realizó análisis bivariado mediante regresión logística no condicional entre cada una de las variables bajo estudio.

Finalmente se elaboraron modelos de regresión logística múltiple para controles, casos y variables sociodemográficas para controlar factores de confusión. Los estimadores fueron calculados al 95\% de confianza. 


\section{RESULTADOS}

Durante la investigación, se reclutaron 110 pacientes del servicio de consulta externa de medicina interna, neumología y urología. A continuación se muestran los resultados:

La proporción del género corresponde 49,09\% al masculino y $50,91 \%$ al femenino.

De los 110 sujetos que participaron (55 casos y 55 controles [no se utilizó método pareado]), 28 (25,45\%) no presentaron sarcopenia, las tres variables de diagnóstico se encontraron en parámetros adecuados, 27 (28,18\%) tuvieron un porcentaje de masa muscular inferior a los parámetros normales pero adecuados valores de fuerza manual y velocidad de marcha, por tanto fueron clasificados en estadio de presarcopenia y se tomaron como controles, 31 sujetos $(28,18 \%)$ se diagnosticaron con sarcopenia al presentar baja masa muscular y al menos una variable de función muscular deficiente (fuerza muscular o rendimiento físico), por último 24 adultos mayores (21,85\%) fueron clasificados en el estadio de sarcopenia grave al presentar baja masa muscular y deterioro en fuerza y rendimiento.

Los controles son más jóvenes que los casos en 2,78 años de edad.

El estado civil y el nivel socioeconómico son características sociodemográficas que se abordaron en el estudio, respecto al estado civil 70,19\% (78 sujetos) tienen pareja, y en el nivel socioeconómico 60\% (66 sujetos) presentan nivel socioeconómico bajo (información obtenida de expedientes clínicos).
El índice de masa corporal (IMC) y el porcentaje de grasa resultaron mayores en los casos que en los controles (Tabla 1), la diferencia es estadísticamente significativa. Respecto a la grasa hay una diferencia del $9 \%$ entre casos y controles (Tabla 2).

La masa muscular es mayor en controles que en casos casi un 7\% ( $P=0,00$; IC95\%:-13,05 a -6,73), se observa mayor fuerza manual y menor tiempo de velocidad de marcha en controles con diferencia estadísticamente significativa (Tabla 2).

Tanto la ingesta de proteína total como la ingesta de proteína expresada en $\mathrm{g} / \mathrm{kg}$ de peso/día son mayores en los controles, destacando que el promedio del consumo de proteína animal es mayor en estos y de proteína vegetal en los casos. La diferencia promedio está en el límite de la significancia estadística para el consumo de proteína animal (Tabla 2).

Respecto a la actividad física, no se halló diferencia estadísticamente significativa entre casos y controles (Tabla 2).

El modelo de regresión logística explica en un 37\% la presencia de sarcopenia, observándose que por cada año de escolaridad se reduce el riesgo un 17\% (RM=0,83; IC95\%:0,73 a 0,94$)$, por cada unidad del porcentaje de grasa se incrementa el riesgo un 20\% ( $\mathrm{RM}=1,20$; IC95\%:1,11 a 1,30), por cada año de edad se incrementa el riesgo un $8 \%(\mathrm{RM}=1,08$; IC95\%:1,00 a 1,17) y por cada gramo de proteína total ingerida se reduce el riesgo un 3\% ( $R M=0,97 ; \mathrm{IC} 95 \%: 0,95$ a 1,00) (Tabla 3).

Tabla 1. Correlación de género, variables sociodemográficas (estado civil y nivel socioeconómico) e IMC con la presencia de sarcopenia.

\begin{tabular}{|c|c|c|c|c|}
\hline Variable & Controles & Casos & $\mathbf{X i}^{2}$ & Pearson \\
\hline Género* & $\begin{array}{l}17 \text { mujeres } \\
38 \text { hombres }\end{array}$ & $\begin{array}{l}39 \text { mujeres } \\
16 \text { hombres }\end{array}$ & 17,6058 & 0,000 \\
\hline Estado Civil* & $\begin{array}{l}44 \text { con pareja } \\
11 \text { sin pareja }\end{array}$ & $\begin{array}{l}34 \text { con pareja } \\
21 \text { sin pareja }\end{array}$ & 4,4051 & 0,036 \\
\hline Nivel socio-económico* & $\begin{array}{c}40 \text { bajo } \\
14 \text { medio } \\
1 \text { alto }\end{array}$ & $\begin{array}{l}26 \text { bajo } \\
21 \text { medio } \\
1 \text { alto }\end{array}$ & 7,6364 & 0,022 \\
\hline $\mathrm{IMC} *$ & $\begin{array}{c}8 \text { Normopeso } \\
18 \mathrm{SP} \\
22 \mathrm{OBI}\end{array}$ & $\begin{array}{c}12 \text { Normopeso } \\
30 \mathrm{SP} \\
8 \mathrm{OB} \mathrm{I}\end{array}$ & 11,3333 & 0,023 \\
\hline
\end{tabular}

(*)Variable con diferencia estadísticamente significativa 
Tabla 2. Diferencia de medias entre casos y controles, respecto a factores de riesgo para sarcopenia.

\begin{tabular}{|c|c|c|c|c|c|c|}
\hline Variable & $\begin{array}{c}\text { Media } \\
\text { de Controles }\end{array}$ & $\begin{array}{c}\text { Media } \\
\text { de Casos }\end{array}$ & $\begin{array}{l}\text { Diferencia } \\
\text { de Medias }\end{array}$ & $\begin{array}{l}\text { Desviación } \\
\text { Estándar }\end{array}$ & $\begin{array}{c}\text { Intervalos } \\
\text { de confianza 95\% }\end{array}$ & $\mathbf{P}$ \\
\hline Porcentaje de grasa corporal* & 30,38182 & 40,27455 & $-9,892727$ & 1,595451 & $-13,05519 /-6,730267$ & 0,0000 \\
\hline Porcentaje de masa muscular* & 38,55091 & 31,16909 & 7,381818 & ,8133472 & 5,769623 / 8,99401 & 0,0000 \\
\hline Fuerza de presión manual $(\mathrm{kg})^{*}$ & 28,48182 & 14,99818 & 13,48364 & 1,33284 & 10,84172 / 16,12556 & 0,0000 \\
\hline Velocidad de la marcha $(\mathrm{m} / \mathrm{s})^{*}$ & 1,251636 & 8498182 & ,4018182 & 0619255 & ,2790712/,5245652 & 0,0000 \\
\hline Proteína total en gramos* & 58,58491 & 47,17091 & 11,414 & 3,945529 & 3,593277 / 19,23472 & 0,0046 \\
\hline Proteína animal en gramos* & 28,92436 & 22,86936 & 6,11 & 3,121913 & -0781724 / 12,29817 & 0,0529 \\
\hline Proteína vegetal en gramos* & 29,55273 & 24,35236 & 5,200364 & 2,279344 & ,6823085 / 9,718419 & 0,0245 \\
\hline g de proteína/kg de peso/día & 8067273 & ,7034545 & 1032727 & ,0628339 & -,021275 / 2278204 & 0,1032 \\
\hline MET & 9,343818 & 8,275273 & 1,068545 & 8036446 &,- 5244175 / 2,661508 & 0,1864 \\
\hline
\end{tabular}

(*) Variable con diferencia estadísticamente significativa

Tabla 3. Resultado del análisis de regresión logística.

\begin{tabular}{l|c|c|c|c|c}
\multicolumn{1}{c|}{ Variable } & $\begin{array}{c}\text { Razón de } \\
\text { momios }\end{array}$ & $\begin{array}{c}\text { Desviación } \\
\text { estándar }\end{array}$ & $\mathbf{Z}$ & $\mathbf{P}>|\mathrm{z}|$ & $\begin{array}{c}\text { Intervalos } \\
\text { de confianza 95\% }\end{array}$ \\
\hline Escolaridad* &, 8368216 &, 0527482 & $-2,83$ & 0,005 &, $7395682 /, 9468639$ \\
Porcentaje de grasa* & 1,207765 &, 0485326 & 4,70 & 0,000 & $1,116293 / 1,306734$
\end{tabular}

$(*)$ Variable con diferencia estadísticamente significa-

\section{DISCUSIÓN}

Se consideraron cuidadosamente los criterios diagnósticos para clasificar casos y controles. Tales criterios son bien aceptados por el EWGSOP y existen suficientes pruebas científicas que lo sustentan. Por consiguiente el $28,18 \%$ de los sujetos con presarcopenia fueron clasificados como controles, ya que la sarcopenia es un síndrome que afecta la cantidad de músculo y su funcionalidad, porque no existe una correlación lineal entre ambos ${ }^{3}$. Mientras la fuerza y el rendimiento sean adecuados para conservar la función, los factores de riesgo de caídas, fracturas y discapacidad se reducen.

El proceso de envejecimiento es un factor de riesgo primario para la sarcopenia. En esta investigación se observa que en promedio los controles son más jóvenes que los casos en 2,78 años.

Respecto al IMC y composición corporal se encontró que los casos tienen mayor IMC y grasa que los controles. Durante el envejecimiento aumenta la grasa y disminuye la masa muscular, lo que en este estudio se confirma porque el porcentaje de masa muscular es mayor en los controles casi un $7 \%$.

En cuanto a la función muscular, los controles presentaron mayor fuerza y menor tiempo de marcha. El deterioro de estos factores aumenta el riesgo de caídas e ingravidez en el paciente geriátrico ${ }^{16}$.

De acuerdo con el objetivo principal del estudio, se determinó la ingesta de proteína total al día y se calculó en $\mathrm{g} / \mathrm{kg}$ de peso/día, la diferencia en ambas es ligeramente mayor en los controles que en los casos. 
El promedio de consumo de proteína animal es mayor en los controles y el consumo de proteína vegetal es mayor en Ios casos, quizá atribuible a que el nivel socioeconómico es bajo en $31,81 \%$ de los sujetos y repercute en la accesibilidad de alimentos, limitando la ingesta de proteínas de alto valor biológico y aminoácidos para síntesis muscular. Esto incrementa el riesgo de problemas de salud asociados a la edad como sarcopenia, osteoporosis y alteraciones del sistema inmunológico $9,17-19$.

Jürgen Bauer, en el artículo PROT-AGE menciona que la calidad y la carga proteica de cada comida influyen en la síntesis, se recomiendan 25 a $30 \mathrm{~g}$ de proteína por comida, que incluya 2,5 a $2,8 \mathrm{~g}$ de leucina para mantener la funcionalidad $^{8}$.

Suficiente proteína, aminoácidos esenciales y ejercicio mejoran la sensibilidad de nutrientes y hormonas ${ }^{20-25}$.

No se encontró diferencia estadísticamente significativa en actividad física entre casos y controles debido a la homogeneidad de MET consumidos en 24 horas, porque la mayoría de los casos y los controles no realizan ejercicio físico de manera regular y su actividad física es ligera.

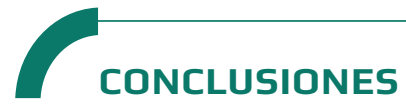

La ingesta proteica de alto valor biológico es un factor protector contra sarcopenia, ya que por cada gramo se reduce el riesgo un $3 \%$. Por el contrario, la excesiva acumulación de grasa corporal es un importante factor de riesgo que debe seguirse investigando dado que la obesidad es un problema grave de salud pública.

\section{$\longrightarrow$ \\ CONFLICTO DE INTERESES}

Los autores expresan que no hay conflictos de intereses al redactar el manuscrito.

\section{FINANCIACIÓN}

Esta investigación forma parte del sistema de becas otorgadas a programas de posgrado avalados por el Consejo Nacional de Ciencia y Tecnología (CONACYT).

\section{BIBLIOGRAFÍA}

(1) Baumgartner RN, Koehler KM, Gallagher D, Romero L, Heymsfield SB, Ross RR, et al. Epidemiology of sarcopenia among the elderly in New Mexico. Am J Epidemiol. 1998; 147(8): 755-63.

(2) Melton L, Khosla S, Crowson CS, O'Connor MK, O'Fallon WM, Riggs BL. Epidemiology of sarcopenia. J Am Geriatr Soc. 2000; 48(6): 625-30.

(3) Cruz-Jentoft AJ, Baeyens JP, Bauer JM, Boirie Y, Cederholm T, Landi $F$, et al. Sarcopenia: European consensus on definition and diagnosis: Report of the European Working Group on Sarcopenia in Older People. Age Ageing. 2010; 39(4): 412-23.

(4) Urteaga C, Ramos RI, Atalah E. Validación del criterio de evaluación nutricional global del adulto mayor. Rev Med Chil. 2001; 129(8): 871-6.

(5) Zavala-González M, Posada-Arévalo S, Cantú-Pérez R. Dependencia funcional y depresión en un grupo de ancianos de Villahermosa, México. Arch Med Fam. 2010; 12(4): 116-26.

(6) Sullivan DH, Patch GA, Walls RC, Lipschitz DA. Impact of nutrition status on morbidity and mortality in a select population of geriatric rehabilitation patients. Am J Clin Nutr. 1990; 51(5): 749-58.

(7) Sullivan DH, Sun S, Walls RC. Protein-energy undernutrition among elderly hospitalized patients: a prospective study. JAMA. 1999; 281(21): 2013-9.

(8) Bauer J, Biolo G, Cederholm T, Cesari M, Cruz-Jentoft AJ, Morley $\mathrm{JE}$, et al. Evidence-based recommendations for optimal dietary protein intake in older people: a position paper from the PROTAGE Study Group. J Am Med Dir Assoc. 2013; 14(8): 542-59.

(9) Rand WM, Pellett PL, Young VR. Meta-analysis of nitrogen balance studies for estimating protein requirements in healthy adults. Am J Clin Nutr. 2003; 77(1): 109-27.

(10) Rieu I, Balage M, Sornet C, Debras E, Ripes S, RochonBonhomme $C$, et al. Increased availability of leucine with leucine-rich whey proteins improves postprandial muscle protein synthesis in aging rats. Nutrition. 2007; 23(4): 323-31.

(11) Katsanos CS, Kobayashi H, Sheffield-Moore M, Aarsland A, Wolfe RR. Aging is associated with diminished accretion of muscle proteins after the ingestion of a small bolus of essential amino acids. Am J Clin Nutr. 2005; 82(5): 1065-73.

(12) González-Torres L, Téllez-Valencia A, Sampedro J, Nájera H. Las proteínas en la nutrición. RESPYN [revista en internet] 2007 [consulta: 19/03/2015];8(2). Disponible en: http://www. respyn.uanl.mx/viii/2/ensayos/proteinas.htm

(13) Pope AM, Tarlov AR. Disability in America: Toward a National Agenda for Prevention. Washington, DC: National Academy Press; 1991.

(14) Evans W. Functional and metabolic consequences of sarcopenia. J Nutr. 1997; 127(5 Suppl): 998S-1003S. 
(15) Chávez J, Lozano M, Lara A, Velázquez O. La actividad física y el deporte en el adulto mayor: bases fisiológicas. 2a ed. México: Masson Doyma México; 2004.

(16) Boyle PA, Buchman AS, Wilson RS, Bienias JL, Bennett DA. Physical activity is associated with incident disability in community-based older persons. ] Am Geriatr Soc. 2007; 55(2): 195-201.

(17) Cederholm TE, Bauer JM, Boirie Y, Schneider SM, Sieber CC, Rolland Y. Toward a definition of sarcopenia. Clin Geriatr Med. 2011; 27(3): 341-53.

(18) de Souza Genaro P, Martini LA. Effect of protein intake on bone and muscle mass in the elderly. Nutr Rev. 2010; 68(10): 61623.

(19) Castaneda C, Dolnikowski GG, Dallal GE, Evans W], Crim MC. Protein turnover and energy metabolism of elderly women fed a low-protein diet. Am J Clin Nutr. 1995; 62(1): 40-8.

(20) Timmerman KL, Dhanani S, Glynn EL, Fry CS, Drummond MJ, Jennings $\mathrm{K}$, et al. A moderate acute increase in physical activity enhances nutritive flow and the muscle protein anabolic response to mixed nutrient intake in older adults. Am J Clin Nutr. 2012; 95(6): 1403-12.
(21) Cermak NM, Res PT, de Groot LCPGM, Saris WHM, van Loon LC. Protein supplementation augments the adaptive response of skeletal muscle to resistance-type exercise training: a metaanalysis. Am J Clin Nutr. 2012; 96(6): 1454-64.

(22) Fujita S, Rasmussen BB, Cadenas JG, Drummond MJ, Glynn EL, Sattler FR, et al. Aerobic exercise overcomes the age-related insulin resistance of muscle protein metabolism by improving endothelial function and Akt/mammalian target of rapamycin signaling. Diabetes. 2007; 56(6): 1615-22.

(23) Yang Y, Churchward-Venne TA, Burd NA, Breen L, Tarnopolsky MA, Phillips SM. Myofibrillar protein synthesis following ingestion of soy protein isolate at rest and after resistance exercise in elderly men. Nutr Metab. 2012; 9(1): 57.

(24) Yang Y, Breen L, Burd NA, Hector AJ, Churchward-Venne TA, Josse $A R$, et al. Resistance exercise enhances myofibrillar protein synthesis with graded intakes of whey protein in older men. $\mathrm{Br}$ ] Nutr. 2012; 108(10): 1780-8.

(25) Volpi E, Mittendorfer B, Rasmussen BB, Wolfe RR. The response of muscle protein anabolism to combined hyperaminoacidemia and glucose-induced hyperinsulinemia is impaired in the elderly. J Clin Endocrinol Metab. 2000; 85(12): 4481-90. 